\title{
Selektiv sättigbare organische Farbstoffe als optische Schalter - optische Impulsverstärker
}

\author{
Dieter Röss \\ Mitteilung aus dem Zentral-Laboratorium der Siemens \& Halske AG., München
}

(Z. Naturforschg. 20 a, 696-700 [1965] ; eingegangen am 24. Februar 1965)

\begin{abstract}
Die Absorption organischer Farbstofflösungen kann durch monochromatisches Licht selektiv in einem gegen die Linienbreite sehr kleinen Spektralbereich $\delta v / \Delta v<10^{-5}$ gesättigt werden. Eine Reihe neuer bei der Emissionsfrequenz von Rubinlasern sättigbarer Substanzen wird beschrieben.

Die Empfindlichkeit selektiv sättigbarer Schalter kann so weit gesteigert werden, daß damit gesperrte Laseroszillatoren durch Fremdlicht geringer Leistung ausgelöst werden können. Die Eignung eines solchen optischen Impulsverstärkers als Quantenzähler und als logisches Element wird diskutiert.
\end{abstract}

\section{Selektive Sättigung}

Absorber mit einer vom Lichtfluß abhängigen Transmission können als passive, schnelle Schalter für Licht verwendet werden. Zumindestens bei den dafür geeigneten organischen Farbstofflösungen wird die Transmission $T$ durch optisches Pumpen des Absorptionsübergangs geändert. Bei verschwindend kleinem Lichtfluß gilt:

$$
T_{0}=e^{-\alpha(v) l}=e^{-\sigma(v) N_{0} l} .
$$

Dabei ist $\sigma(v)$ der Absorptionswirkungsquerschnitt, $N_{0}=N_{1}$ die Zahl der Moleküle pro $\mathrm{cm}^{3}$ im Grundzustand $E_{1}, \alpha(v)=\sigma(v) N_{1}$ der Absorptionskoeffizient und $l$ die Absorptionslänge.

Durch den Absorptionsprozeß gehen $N_{2}$ Moleküle in den Zustand $E_{2}$ über; für die veränderte Transmission gilt bei einer homogen verbreiterten Linie:

$$
T=e^{-\sigma(v)\left(N_{1}-N_{2}\right) l} .
$$

Bei hohem Lichtfluß geht der Übergang in Sättigung; die Transmission geht gegen 1 :

$$
N_{2}=N_{1}=N_{0}, \quad T=1 .
$$

Der gesättigte Übergang kommt nach Abschalten der Lichtquelle mit der Zeitkonstanten $\tau$ wieder ins thermische Gleichgewicht. Wenn das sättigende Licht als Impuls der Dauer $t \ll \tau$ zugeführt wird, dann ist die Sättigungsenergie gleich

$$
E_{\mathrm{s}}=\frac{1}{2} N_{0} h v .
$$

1 P. P. Sorokin, I. J. Luzzi, J. R. Lankard u. G. D. Pettit, IBM-J. 8, 182 [1964].

2 G. Bret u. F. Gires, Appl. Phys. Letters 4, 175 [1964].

3 P. SснӓғеR u. W. Sснмidt, Z. Naturforschg. 19 a, 1019 [1964].
Die obige Abschätzung führt zu der Annahme, daß eine Sättigung der für solche Zwecke bisher verwendeten Farbstoffe wie Methylenblau, Chlorophyll, Viktoriablau B, den Cryptocyaninen und den Phtalocyaninen nur bei sehr hohen Lichtflüssen möglich ist, wie sie etwa in Rubinlasern vorliegen ${ }^{1-4}$.

In Wirklichkeit sind die notwendigen Sättigungsenergien sehr viel niedriger als aus Gl. (4) hervorgehen würde, weil die Sättigung spektral selektiv ist $^{5}$. Die Absorptionslinien sind inhomogen verbreitert und nur diejenigen Atome müssen zu einer selektiven Sättigung angeregt werden, die innerhalb der Sättigungsbandbreite $\delta v$ liegen (Abb. 1). Mit $g(v)$ als normierter Linienform [ $\left.\int g(v) \mathrm{d} v=1\right]$ beträgt die Sättigungsenergie nur

$$
\frac{E_{\mathrm{s}}}{h v}=\frac{N_{0}}{2} g(v) \delta v \approx \frac{N_{0}}{2} \frac{\delta v}{\Delta v} \frac{g(v)}{g\left(v_{0}\right)} .
$$

Dabei ist $\Delta v$ die äquivalente Breite der Absorptionslinie und $v_{0}$ die Frequenz der Linienmitte.

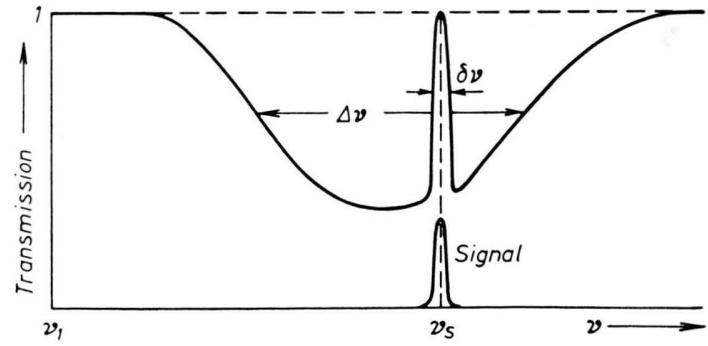

Abb. 1. Selektive Sättigung einer Absorptionslinie durch ein quasimonochromatisches Lichtsignal.

4 P. Kafalas, J. I. Masters u. E. M. E. Murray, J. Appl. Phys. 35, 2349 [1964].

5 D. Röss, "Laser"; Vortrag auf der Elektroniktagung im Rahmen der FERA Zürich, August 1964, Bull. Schweiz. elektrotechn. Verein, im Druck. 
Wenn sättigbare Absorber als Schalter in Riesenimpuls-Lasern verwendet werden ${ }^{1-4}$, dann muß gelten:

$$
\begin{aligned}
& V(t) R T_{0}\left\{\begin{array}{lll}
<1 & \text { für } t<t_{1}, \\
\approx 1 & \text { für } t=t_{1},
\end{array}\right. \\
& V R \cdot 1 \gg 1 \text { für } t=t_{1} \text {. }
\end{aligned}
$$

$V$ ist die Verstärkung pro Durchgang, $R$ die mittlere Spiegelreflexion. Nach (6) erreicht der Laser zum Zeitpunkt $t_{1}$ seine Schwelle bei unveränderter Anfangsabsorption $T_{0}$ und bildet dann durch Sättigung $T_{0} \rightarrow 1$ einen Riesenimpuls aus. Der Lichtfluß im Resonator, gegeben durch die spontane Emission des Lasermaterials, ist unterhalb der Schwelle so klein, daß die Linie als homogen verbreitert wirkt. Die Sperrwirkung des Absorbers ist zu berechnen nach Gl. (1). Für die Zahl der notwendigen Moleküle folgt:

$$
N_{0}=\frac{-\ln T_{0}}{\sigma(v) l}=\frac{a_{0}}{\sigma(v)}=\frac{a_{0}}{\sigma\left(v_{0}\right)} \frac{g\left(v_{0}\right)}{g(v)} .
$$

Für die selektive Sättigungsenergie ergibt sich:

$$
\frac{E_{\mathrm{S}}}{h v}=\frac{a_{0}}{\sigma\left(v_{0}\right)} \cdot \frac{\delta v}{\Delta v} \frac{\text { Quanten }}{\mathrm{cm}^{2}} .
$$

Die Sättigungsenergie bei gegebener Anfangsabsorption $\alpha_{0}$ ist unabhängig davon, ob die Signalfrequenz in der Linienmitte liegt oder an der Linienflanke. Dies gilt nur, solange die Moleküle innerhalb der Linie alle gleichwertig sind. Man wird in der Praxis bestrebt sein, die Linienmitte etwa mit der Signalfrequenz zur Deckung zu bringen, um mit kleinen Konzentrationen arbeiten zu können. Die wichtigen Größen sind der absolute Wirkungsquerschnitt und das Verhältnis der Sättigungsbandbreite $\delta v$ zur gesamten Linienbreite $\Delta v$. Nach Gl. (8) ist ein Material mit großer Linienbreite $\Delta v$ einem anderen mit schmaler Linie bei gleichem Wirkungsquerschnitt vorzuziehen.

\section{Beobachung selektiver Sättigung an Riesenimpuls-Lasern}

Wir haben den Effekt der selektiven Sättigung bei der Suche nach neuen Farbstoffen für Absorptionsschalter beobachtet ${ }^{5}$. In den ebenen FABRY-PERotResonator eines Rubinlasers (Rubin $7 \mathrm{~mm} \phi, 70 \mathrm{~mm}$ lang) wurde eine $1 \mathrm{~cm}$ lange Absorptionszelle eingebracht. Ohne absorbierenden Farbstoff betrug die Schwellenergie des in einem Rotationsellipsoidspiegel ${ }^{6}$ mit einer Blitzlampe FX42 gepumpten Lasers bei 5\% Auskopplung $110 \mathrm{~W}$ s. Bei $200 \mathrm{Ws}$ war die Verstärkung so hoch, daß der einseitig $100 \%$ reflektierende, auf der anderen Endfläche unvergütete Stab ohne äußeren Spie-

${ }^{6}$ D. Röss, Appl. Optics 3, 259 [1964]. gel seine Schwelle erreichte. In Abb. $2 *$ sind Aufnahmen der zeitlichen Emission bei $190 \mathrm{Ws}$ Pumpenergie aufgezeichnet. Bei der Aufnahme von Abb. 2 a enthielt die Zelle reines Äthanol; die Laserenergie beträgt 0,45 $W_{s}$, die Spitzenleistung in den Relaxationsimpulsen rund $5 \mathrm{~kW}$. In den Aufnahmen $2 \mathrm{~b}-2 \mathrm{f}$ wurde in wachsender Konzentration als absorbierender Farbstoff „Sudanschwarz B“ in der Zelle gelöst. Bereits bei einer visuell nicht erkennbaren Konzentration $(2 b-c)$ steigt die Spitzenleistung um einen Faktor 10; in Abb. $2 \mathrm{f}$ ist sie bei blaßblauer Färbung der Lösung gegenüber 2 a um einen Faktor 400 auf $2 \mathrm{MW}$ erhöht. Die Impulse werden mit wachsender Anfangsabsorption erwartungsgemäß seltener und annähernd periodisch. Die unterschiedliche Höhe erklärt sich in Abb. $2 \mathrm{~d}-\mathrm{e}$ daraus, $\mathrm{da} ß$ während des Pumpimpulses die Inversion im $\mathrm{Ru}$ binquerschnitt unterschiedlich schnell ansteigt, so daß bei den späteren Impulsen ein größeres Materialvolumen zur Emission kommt.

Abb. 3 zeigt in einer entsprechenden Aufnahmereihe mit zunehmender Anfangsabsorption das Spektrum der Emission. Als Spektrometer wurde ein Fabry-PerotInterferometer hoher Auflösung mit dielektrischen Spiegeln von 95\% Reflexion verwendet. Es sind Ausschnitte aus 2 Ordnungen erkennbar. Der Dispersionsbereich beträgt bei $5 \mathrm{~mm}$ Etalondicke $10 \mathrm{GHz}$. In Abb. 3 a sind die einzelnen axialen Moden des etwa $15 \mathrm{~cm}$ langen Resonators klar aufgelöst. Alle Moden innerhalb der Verstärkungsbandbreite treten auf. Mit wachsender Anfangsabsorption des sättigbaren Farbstoffes „Sudanschwarz B“ beobachtet man immer weniger Moden, wobei einzelne axiale Moden ausgelassen werden. Wenn nur zwei Riesenimpulse emitiert werden, wie in Abb. $2 \mathrm{f}$, dann sind nur zwei Moden angeregt (Abb. $3 \mathrm{~d})$. Bei den vorhergehenden Aufnahmen $3 \mathrm{~b}-\mathrm{c}$ läßt sich zeigen, daß jeder Riesenimpuls in einem Mode schwingt. Die Zahl der Ringe ist identisch mit der Zahl der Riesenimpulse.

Abb. 4 und 5 zeigen eine Bestätigung dieser Beobachtung. Abb. 4 a zeigt eine Emissionsaufnahme, bei der in einem Pumpimpuls mit $190 \mathrm{Ws}$ ein einzelner Riesenimpuls von $4 \mathrm{MW}$ emittiert wird. Abb. $5 \mathrm{~b}$ zeigt das Spektrum der Emission ohne Schalter, 5 a das aus nur einem Ring bestehende Spektrum des Riesenimpulses. Bei Abb. $4 \mathrm{c}$ wurde unter sonst gleichen Bedingungen die Pumpenergie auf $300 \mathrm{Ws}$ erhöht; der Laser emittiert 7 Riesenimpulse (der erste triggert die Aufnahme). Das Spektrum in Abb. 5 c zeigt 7 Frequenzen. $\mathrm{Da} \beta$ die einzelnen Impulse in verschiedenen Moden emittieren, ist eine Folge der thermischen Liniendrift während des Pumpimpulses. Es emittiert jeweils der Mode, der der Linienmitte am nächsten ist.

Die beobachtete Modenselektion entsteht dadurch, daß der der Linienmitte am nächsten liegende Mode etwas früher seine Schwelle erreicht und exponentiell anwächst als die anderen Moden. Die von ihm verursachte selektive Sättigung der Absorption führt zu einer so starken Bevorzugung, daß die anderen Moden in der Aufbauzeit des resultierenden Riesenimpulses keine merkliche Leistung erreichen.

* Abb. 2-5 auf Tafel S. 698 a, b. 
Aus den Aufnahmen von Abb. 5 können wir entnehmen, daß die Sättigungsbandbreite einer monochromatischen Linie kleiner ist als der Abstand axialer Moden in unserem Resonator, also

$$
\delta v<1 \mathrm{GHz}=10^{9} \mathrm{~s}^{-1} .
$$

Bei einer Bandbreite $\Delta v$ der absorbierenden Substanzen von typischerweise $10^{14} \mathrm{~s}^{-1}$ ist der Bruchteil

$$
\delta v / \Delta v<10^{-5} .
$$

Der Schalteffekt ist sehr viel empfindlicher als bei einer Sättigung der ganzen Linienbreite.

Bei einer Erhöhung der Pumpenergie auf $600 \mathrm{Ws}$ und passender Steigerung von $T_{0}$ konnten wir mit dem verwendeten Rubin bei 84\% Auskopplung (Glas-Etalon als Reflektor ${ }^{7}$ ) Einzelimpulse von 0,53 Ws Energie erzeugen. Die Impulsdauer war dabei kleiner als $12 \mathrm{ns,}$ die kürzeste Impulszeit, die auf dem zur Verfügung stehenden Oszillographen auflösbar war. Bei diesen Spitzenleistungen von über $100 \mathrm{MW}$ wurde der Rubin in etwa 10 Impulsen zerstört. Die Belastungsgrenze scheint bei $100 \mathrm{MW} / \mathrm{cm}^{2} \mathrm{zu}$ liegen.

\section{Neue sättigbare Farbstoffe}

Wir haben eine größere Zahl von neuen sättigbaren Farbstoffen gefunden. Unsere Beobachtungen zeigten, daß jeder der untersuchten organischen Farbstoffe, der bei der Rubin-Linie überhaupt merklich absorbiert, auch in einem Laserresonator sättigbar ist.

Die Linienmitte kann durch die Wahl des Lösungsmittels in weiten Grenzen verschoben werden. In Übereinstimmung mit den obigen Überlegungen ist die genaue Anpassung der Linienmitte an die Signalfrequenz nicht wichtig.

Durch das Lösungsmittel wird die Relaxationszeit $\tau$ beeinflußt. Damit der Stoff in einem Laser- resonator sättigbar ist, darf $\tau$ nicht sehr klein gegen die Anstiegszeit des Riesenimpulses von etwa $10^{-8}$ bis $10^{-7} \mathrm{~s}$ sein. Bei einer Reihe von Farbstoffen beobachteten wir, daß bei nahezu unveränderter Linienform in verschiedenen Lösungsmitteln als Folge unterschiedlicher Relaxationszeit eine Schaltfunktion nur in bestimmten Lösungsmitteln auftrat, wobei teilweise Mischungen von Lösungsmitteln notwendig waren.

Die Sättigungsenergie ist bei allen brauchbaren Farbstoffen so gering, daß die Wahl des speziellen Farbstoffes für Riesenimpuls-Laser von sekundären Faktoren abhängig gemacht werden kann.

Neue Farbstoffe, die bereits in geringster Konzentration bei $6943 \AA$ als Schalter verwendet werden können, sind in Tab. 1 aufgeführt. In Tab. 2 sind Farbstoffe zusammengefaßt, die bei höherer Konzentration als Schalter geeignet sind.

In den Abb. 6-8 sind Absorptionskurven einiger für die Rubinlinie sehr gut geeigneter Farbstoffe in verschiedenen Lösungsmitteln aufgezeichnet. Die Konzentrationen liegen zwischen $10^{16}-10^{17}$ Molekülen $/ \mathrm{cm}^{3}$ für eine Anfangsabsorption $\alpha_{0} \sim 1 \mathrm{~cm}^{-1}$.

\begin{tabular}{|l|c|c|c|}
\hline & In Wasser & In Alkohol & $\begin{array}{c}\text { Mole- } \\
\text { kular- } \\
\text { gewicht }\end{array}$ \\
\hline Brillantkresylblau & unlöslich & + & 318 \\
Malachitgrün & + & + & 365 \\
Wasserblau & + & - & 800 \\
Azur II & + & - & 312 \\
Brillantgrün & + & unlöslich & 483 \\
Anilinblau & + & unlöslich & 800 \\
Eriochromschwarz & + & unlöslich & 518 \\
\hline
\end{tabular}

Tab. 2. Farbstofflösungen, die bei höherer Konzentration für

\begin{tabular}{|c|c|c|c|c|}
\hline & In Wasser & In Äthanol & $\begin{array}{c}\text { Lösungsmittel für } \\
\text { Linienmitte nahe } 6943 \AA\end{array}$ & $\begin{array}{l}\text { Molekular- } \\
\text { gewicht }\end{array}$ \\
\hline $\begin{array}{l}\text { Sudanschwarz B } \\
\text { Janusgrün } \\
\text { Methylengrün } \\
\text { Nachtblau } \\
\text { Naphtholblau } \\
\text { Naphtholgrün } \\
\text { Filterblaugrün } \\
\text { Toluidingrün } \\
\text { Toluidinblau }\end{array}$ & $\begin{array}{l}\text { unlöslich } \\
+ \\
+ \\
+ \\
- \\
- \\
- \\
- \\
-\end{array}$ & $\begin{array}{c}+ \\
+ \\
\text { unlöslich } \\
+ \\
+ \text {, Spur } \mathrm{H}_{2} \mathrm{O} \\
+ \text {, Spur } \mathrm{H}_{2} \mathrm{O} \\
+ \text {, Spur } \mathrm{H}_{2} \mathrm{O} \\
+ \text {, Spur } \mathrm{H}_{2} \mathrm{O} \\
+ \text {, Spur } \mathrm{H}_{2} \mathrm{O}\end{array}$ & $\begin{array}{l}\text { Essigsäure } \\
\text { Glyzerin } \\
\text { Äthanol }+10 \% \mathrm{H}_{2} \mathrm{O} \\
\text { Äthanol }+10 \% \mathrm{H}_{2} \mathrm{O} \\
\text { Äthanol }+10 \% \mathrm{H}_{2} \mathrm{O} \\
\text { Äthanol }+10 \% \mathrm{H}_{2} \mathrm{O} \\
\text { Äthanol, Formalin, } \\
\text { Aceton, Methylcyclopentan, } \\
\text { Tetrahydrofuran, Cyclo- } \\
\text { hexan, Amylmethylketon, } \\
\text { Essigsäurebutylester }\end{array}$ & $\begin{array}{l}457 \\
511\end{array}$ \\
\hline $\begin{array}{l}\text { Eriochromschwarz T } \\
\text { Nilblauchlorid }\end{array}$ & $\bar{t}$ & $\begin{array}{l}+ \\
+\end{array}$ & & $\begin{array}{l}461 \\
353\end{array}$ \\
\hline
\end{tabular}
$6940 \AA$ als sättigbare Schalter verwendet werden können.

Tab. 1. Farbstofflösungen, die für die Rubinlinie als sättigbare Schalter besonders geeignet sind.

7 D. Röss, Proc. IEEE 52, 196 [1964]. 
D. Röss, Selektiv sättigbare organische Farbstoffe als optische Schalter - optische Impulsverstärker (S. 696).
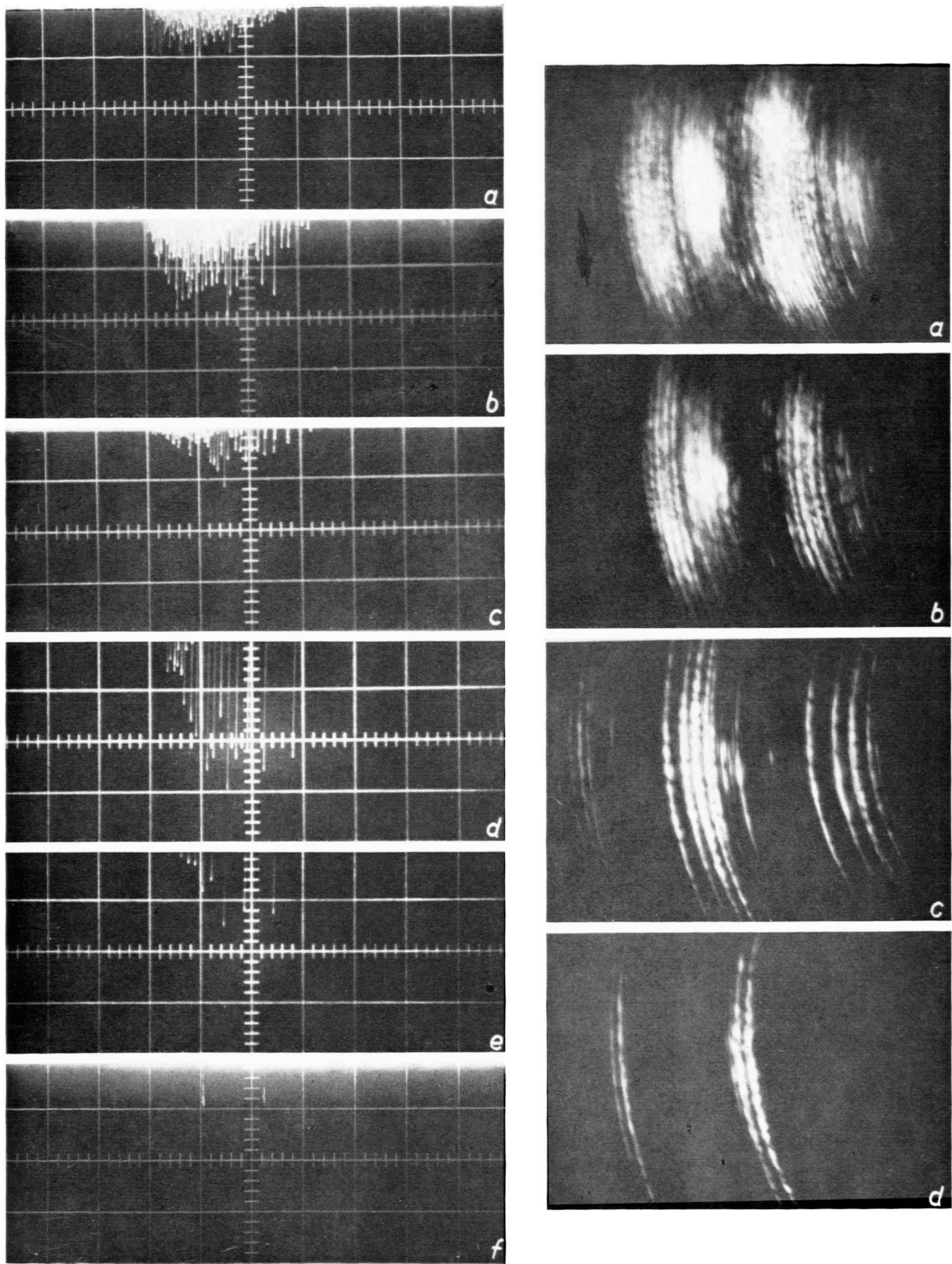

Abb. 2. Emission eines Rubinlasers mit wachsender Anfangsabsorption der Schaltzelle a $-\mathrm{f}$.

Amplitude \begin{tabular}{cccccc} 
a & b & c & d & e & f \\
\hline$\times 1$ & $\times 1$ & $\times 10$ & $\times 10$ & $\times 100$ & $\times 400$
\end{tabular}

Abb. 3. Spektrum der Emission eines Rubinlasers mit wachsender Anfangsabsorption der Schaltzelle a-d. Fabry-PerotAufnahmen; 5-mm-Etalon; Ausschnitte aus zwei Ordnungen. 

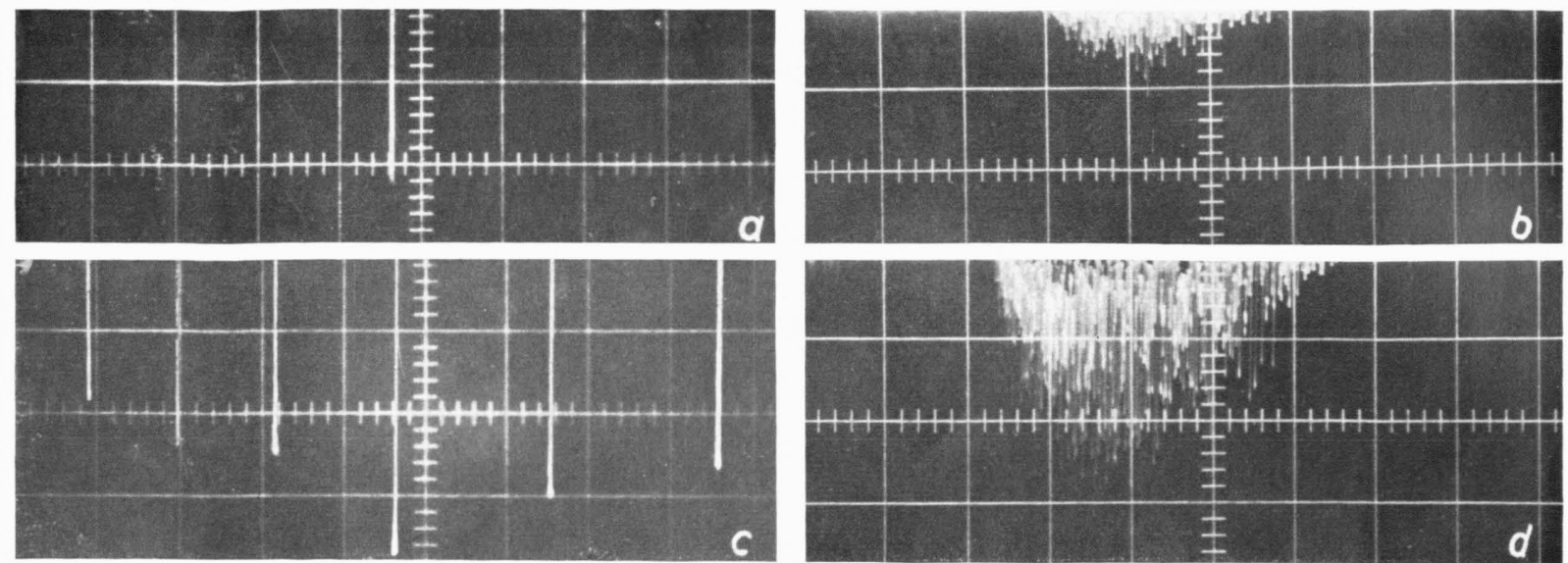

Abb. 4. Riesenimpulse und normale Emission (100 $\mu \mathrm{s} /$ Einheit).

a und c mit Schalter (a $190 \mathrm{Ws}$, c $300 \mathrm{Ws}$ ).

b und d ohne Schalter (b $190 \mathrm{Ws}, \mathrm{d} 300 \mathrm{Ws}$ ).
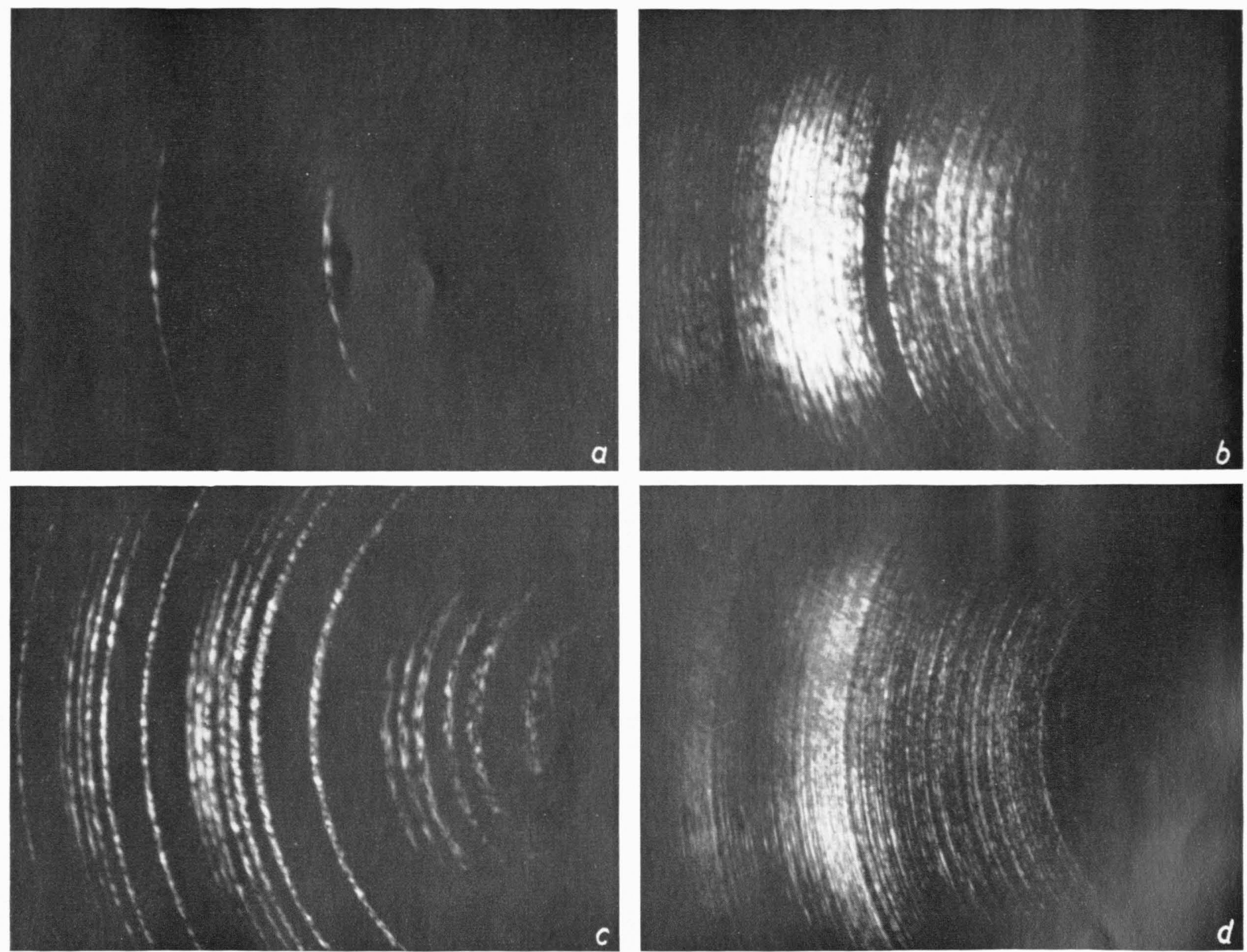

Abb. 5. Emissionspektren zu Abb. 4; jeder Ring entspricht einem axialen Mode. a, b, c, d wie in Abb. 4. F nahmen; 5-mm-Etalon. 


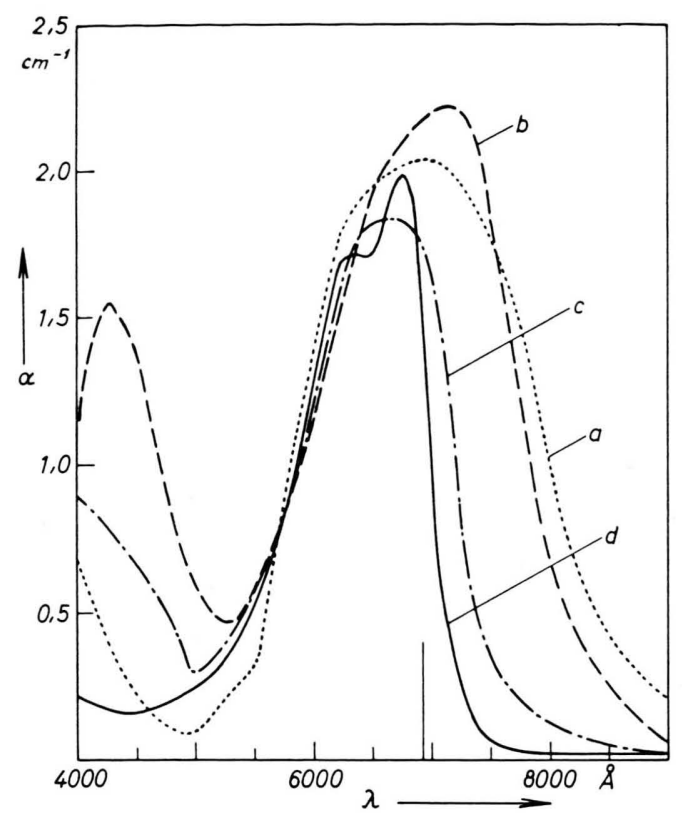

Abb. 6. Absorptionslinien einiger sättigbarer Farbstoffe mit maximaler Absorption bei der Rubinlinie; Lösungsmittel Äthanol $+10 \% \mathrm{H}_{2} \mathrm{O}$. a) Naphtholgrün, $3,4 \cdot 10^{16} \mathrm{~cm}^{-3}$; b) Filterblaugrün, $5 \mathrm{mg} / 100 \mathrm{~cm}^{3}$; c) Toluidingrün, $5 \mathrm{mg} /$ $100 \mathrm{~cm}^{3}$; d) Toluidinblau, $5 \mathrm{mg} / 100 \mathrm{~cm}^{3}$.

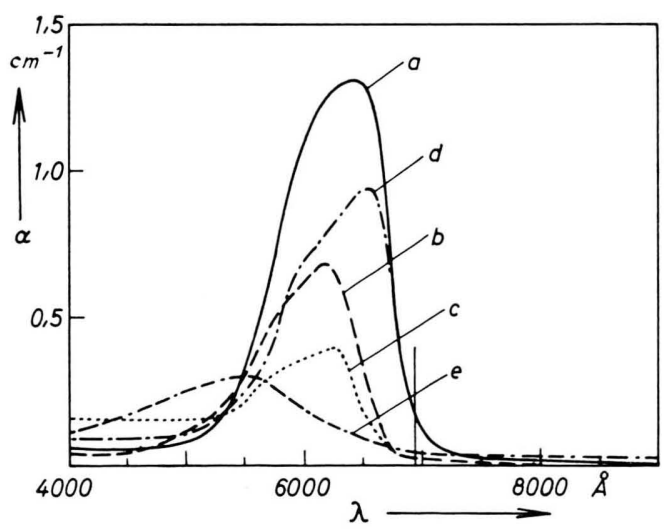

Abb. 7. Absorptionslinien einiger in wäßriger Lösung bei der Rubinlinie sättigbarer Farbstoffe mit großer maximaler Absorption. a) Nilblauchlorid, $10^{16} \mathrm{~cm}^{-3}$; b) Nachtblau, $0,5 \mathrm{mg} / 100 \mathrm{~cm}^{3}$; c) Naphtholblau, $10^{16} \mathrm{~cm}^{-3}$; d) Methylengrün, $10^{16} \mathrm{~cm}^{-3}$; e) Eriochromschwarz $\mathrm{T}, 10^{16} \mathrm{~cm}^{-3}$.

\section{Erhöhung der Empfindlichkeit, Quantenzähler Impulsverstärker}

Zur Erhöhung der Empfindlichkeit kann der zu sättigende Querschnitt verringert werden. Bei einem Laser, der im Grundmode schwingt, ist z. B. durch die Anordnung von Abb. 9 a eine Bündelung auf

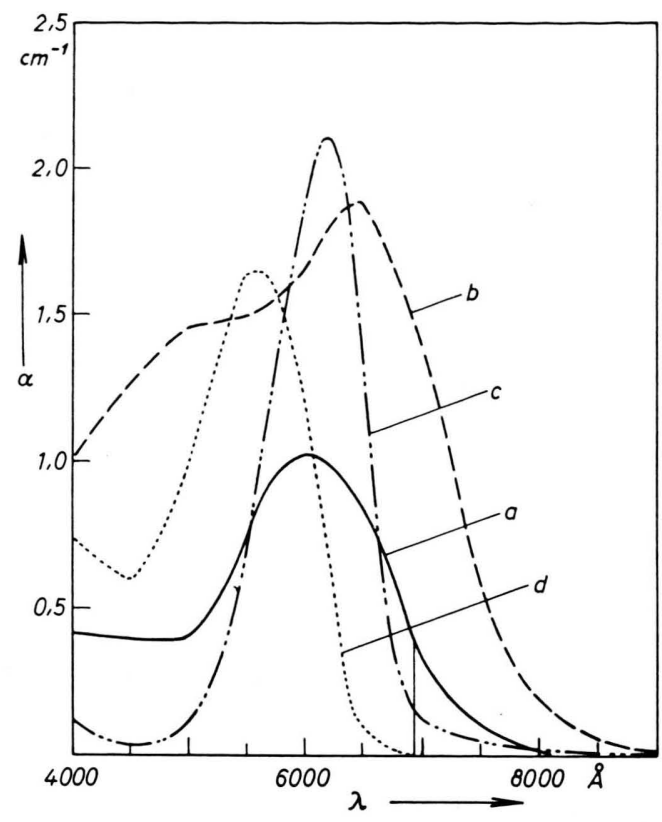

Zu Abb. 8 a: Sudanschwarz B: a) Äthanol, $2 \cdot 10^{16} \mathrm{~cm}^{-3}$; b) Essigsäure, $10^{16} \mathrm{~cm}^{-3}$; c) Glycerin, $10^{16} \mathrm{~cm}^{-3}$; d) Methylcyclopentan, $10^{16} \mathrm{~cm}^{-3}$.

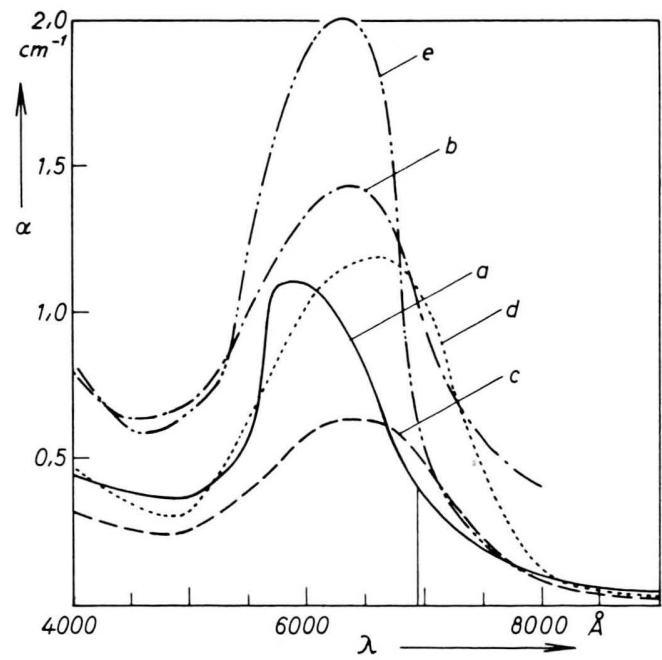

Zu Abb. 8 b: Janusgrün: a) $\mathrm{H}_{2} \mathrm{O}, 10^{16} \mathrm{~cm}^{-3}$; b) Methanol, $10^{16} \mathrm{~cm}^{-8}$; c) Formaldehyd, $10^{16} \mathrm{~cm}^{-3}$; d) Glycerin, $10^{16} \mathrm{~cm}^{-3}$; e) Essigsäure, $10^{16} \mathrm{~cm}^{-3}$.

Abb. 8. Verschiebung der Linie in verschiedenen Lösungsmitteln für Sudanschwarz B (a) und Janusgrün (b) .

einen Querschnitt $Q$ von $\lambda^{2} \approx 5 \cdot 10^{-9} \mathrm{~cm}^{2}$ möglich. Mit $\delta v / \Delta v=10^{-5}$ folgt für die zu einer Sättigung ausreichende Quantenzahl

$$
q=\frac{\alpha_{0}}{\sigma\left(v_{0}\right)} \frac{\delta v}{\Delta v} Q .
$$

Mit $\sigma\left(v_{0}\right) \approx 10^{-16} \mathrm{~cm}^{2}, \delta v / \Delta v=10^{-5}$ erhalten wir

$$
q=\alpha_{0} 500 \text {. }
$$



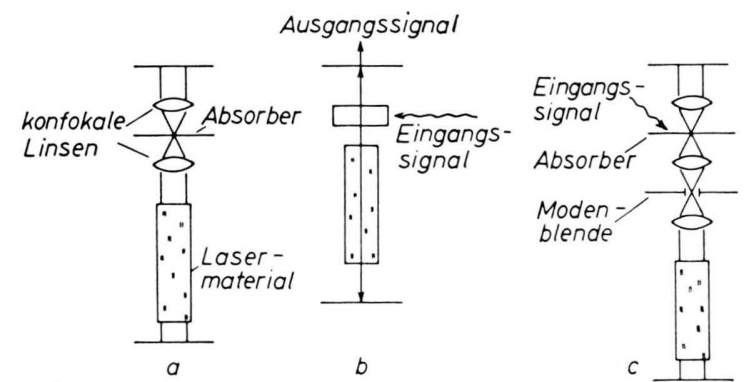

Abb. 9. a) Erhöhung der Empfindlichkeit durch Fokussierung im Resonator. b) Auslösung durch Fremdlicht; optischer Impulsverstärker. c) Empfindlicher Impulsverstärker mit Modenbegrenzung.

Die notwendige Quantenzahl ist bei richtiger Bemessung sehr niedrig. Dies eröffnet die Möglichkeit, den durch einen sättigbaren Absorber gesperrten Laseroszillator zu einem optischen Impulsverstärker zu entwickeln, der durch fremdes Signallicht ausgelöst wird (Abb. 9b). Wir wählen als Anfangsbedingung:

$$
\begin{gathered}
V(t) R T_{0}<1, \\
V\left(t_{1}\right) R T_{\mathrm{s}} \approx 1, \\
V\left(t_{1}\right) R 1>1 .
\end{gathered}
$$

$T_{\mathrm{s}}$ ist die durch das Signallicht zum Zeitpunkt $t_{1}$ erzeugte Transmission. Es ist nicht notwendig, daß das Signallicht eine völlige Sättigung hervorruft. Wir wählen :

$$
\begin{aligned}
V_{0} R T_{0} & =1-\varepsilon, \\
V_{0} R T_{\mathrm{s}} & =1-\varepsilon^{\prime}, \\
V R & =1-\varepsilon^{\prime \prime} .
\end{aligned}
$$

$\varepsilon^{\prime \prime}$ ist die Abweichung der Ringverstärkung $V R$ von der Eins im eingeschwungenen Zustand. Ihr Betrag hängt ab vom Verhältnis der spontanen zur stimulierten Emission pro Mode; in einem Laser guter Modenselektion ist $\varepsilon^{\prime \prime}$ z. B. $10^{-8}$. Für eine Senkung der Quantenzahl unter einen gegen den Gleichgewichtswert verschwindenden Betrag genügt die Wahl von $\varepsilon=10^{-2}$. Wenn das Signal eine Erhöhung der Transmission erzeugt, so da $\varepsilon^{\prime}=\varepsilon^{\prime \prime}$ ist, dann wird der Laser mit Sicherheit ausgelöst. Zum Einschalten des Lasers durch ein äußeres Lichtsignal genügt eine Erhöhung $T_{\mathrm{s}}=T_{0}\left(\varepsilon^{\prime}-\varepsilon\right) \approx 10^{-2} T_{0}$.

Damit ist die nötige Quantenzahl zum Einschalten des Lasers

$$
q=\frac{\ln \left(T_{\mathrm{s}} / T_{0}\right)}{\sigma\left(v_{0}\right)} \frac{\delta v}{\Delta v} Q \approx 5 .
$$

Der Oszillator reagiert auf einzelne Quanten und kann als empfindlicher Quantenzähler verwendet werden. Seine Stabilität ist begrenzt durch die Statistik der spontanen Emission pro Frequenzintervall $\delta v$, die so niedrig wie möglich gehalten werden muß. Abb. 9 c zeigt, wie durch ein Linsensystem im Resonator mit einer Modenblende die spontane Emission optimal begrenzt werden kann.

Wenn Laser als Signallichtquellen verwendet werden, ist die Optimalisierung der Empfindlichkeit wegen des hohen zur Verfügung stehenden Quantenflusses nicht notwendig. Wir haben experimentell einen gesperrten Rubin-Riesenimpulslaser mit der Emission eines zweiten, einfachen Rubinlasers ohne Bündelung des Lichtes auf den Absorber auslösen können.

Der so verwirklichte optische Impulsverstärker hat einige bemerkenswerte Eigenschaften: Das Ausgangssignal ist gerichtet, kohärent und polarisiert, während das Eingangssignal in weiten Grenzen ungerichtet, nichtkohärent und unpolarisiert sein kann. Außerdem überträgt der Verstärker die Signalfrequenz in dem Sinn, daß der Mode innerhalb der Verstärkungsbandbreite schwingt, der der Frequenz des monochromatischen Signallichts am nächsten liegt. Der Verstärker ist nur für Licht in der Verstärkungsbandbreite empfindlich.

Bei Verwendung einer Drei-Niveau-Substanz als Absorber ist eine Frequenzumsetzung zwischen Signallicht und Ausgangslicht möglich, wenn beide ein gemeinsames Niveau besitzen (Abb. 10).

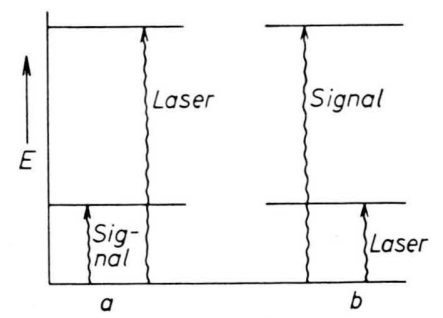

Abb. 10. Frequenzumsetzung in einem schaltbaren Laser, dessen sättigbarer Absorber ein 3-Niveau-System hat.

Der optische Impulsverstärker kann als logisches Element in einem reinen Licht-Datensystem verwendet werden. Wir werden über diese Anwendung an anderer Stelle zusammenhängend berichten. Es sei darauf hingewiesen, daß bei optischen Impulsverstärkern auf Halbleiterbasis mit der Realisierbarkeit von Impulsdauern von $10^{-12} \mathrm{~s}$ und von Impulsrepetitionsfrequenzen von $10^{+11} \mathrm{~s}^{-1}$ gerechnet werden kann.

Fräulein A. Kranefuss und Herrn D. Möckel danke ich für die sorgfältige Messung der Absorptionslinien. 